\title{
ACCURATE STEREOTAXIC LOCALIZATION USING COMPUTERIZED TOMOGRAPHY WITH GEOMETRIC CORRECTION
}

\author{
JiANN-DER LEE ${ }^{\prime}$, ZHONG-XIAN HUANG ${ }^{\prime}$, SHIH-TSENG LEE ${ }^{2}$ \\ 'Department of Electrical Engineering, Chang Gung University, Tao-Yuan \\ ${ }^{2}$ Department of Neurosurgy, Chang Gung Memorial Hospital Tao-Yuan, Taiwan
}

\begin{abstract}
Stereotaxic localization using computerized tomography (CT) is widely employed to guide neurosurgical procedures in critical areas of the brain. Existing methods used two-dimensional (2-D) transform to correct the localization error due to the head ring tilt, but these estimates cannot yield satisfactory results under large tilt angle. Based on three-dimensional (3-D) geometric transformation. a novel approach of correcting localization error from head ring tilt is introduced, resulting in significant improvement in localization accuracy. Experimental results also confirm the good performance of this localization scheme. A powerful stereotactic software system integrated with the proposed method, fusion of brain atlas and CT data, and surgical trajectory planning, has been successfully developed and applied to clinic application for neurosurgery in Chang Gung Memorial Hospital.
\end{abstract}

Biomed Eng Appl Basis Comm, 2002 (October); 14: 189-196.

Keywords: Medical image processing, Stereotaxic localization, Computerized tomography, Geometric correction

\section{INTRODUCTION}

Recently, the spatial resolution of the computerized tomography (CT) scans and magnetic resonance (MR) image has improved to the point that an accurate Cartesian coordinate system can be defined by combining pixel size and scan slice thickness. With these techniques, stereotactic coordinates may be easily calculated for points on CT scans using CT-compatible stereotactic localization systems [1-9]. This improvement allows one to determine the 3-D coordinates of external landmarks or features of a patient's head as identified on sets of image slices. These same external landmarks can then be matched on the patient's head so that the applicable stereotactic coordinates can be

Received: June 12, 2002; Accepted: Oct. 2, 2002

Correspondence: Jiann-Der Lee, Ph. D., Professor

Department of Electrical Engineering Chang Gung University, Tao-Yuan, Taiwan 333, R. O. C.

E-mail: jdlee@mail.cgu.edu.tw established. These techniques also enable the neurosurgeon to move from cumbersome and invasive frame-based stereotactic localization of intracranial structures to less complex localization procedures with the same or better accuracy. In short, the primary benefit of stereotactic surgery is the accuracy with which small lesions can either be made or approached and resected. The resurgence of interest in this field also follows dramatic improvements in radiographic technology.

As described by Kelly [10], stereotactic localization is the process of assigning 3-D stereotactic frame coordinate to an intracranial target structure, which is a point within a normal anatomic structure or a point within a tumor in tumor stereotaxis. In the past, many alternative radiosurgical systems have been developed and judged by the precision with which they local and irradiate a target [4-9]. Winston and Lutz [11] have shown that localization error is the major source of treatment error in radiosurgery. It would therefore be an important task to determine how to minimize the error of CT localization. In order to reduce the error of target localization, several parameters used for CT 
scanning should be selected for maximum accuracy. These parameters with a significant effect on localization error included the scan thickness, interscan spacing (spacing between serial CT scans) and the method of determining fiducial location [12]. This paper will focus on removing the last error source, i.e., fiducial location determination. In general, functional stereotaxic neurosurgery has required definition of the line between the anterior and posterior commissures (so called intercommissural line, AC-PC) as the basis for defining the target site for a procedure such as stereotaxic thalamotomy. More specifically, AC, the anterior commissure, is localized 3-4 mm below the lower border of the foramen of Monro; PC, the posterior commissure, can be seen on the slice immediately superior to the quadrigeminal plate or the upper end of the aqueduct. While axial CT imaging precisely defines the commissures using standard CT software, the planes of the axial images are not usually parallel to the important intercommissural line due to the head ring tilt (or rotation) [5], and the plane of the intercommissural line cannot be predicated from the external anatomy of the skull. Clearly, it is almost impossible for a stereotactic system to provide the advanced functions such as $3 \mathrm{D}$ atlas fusion, surgical trajectory planning, etc. without accurate target location. The accuracy of the localization scheme mainly depends on how efficient the correction strategy used for removing the deviation of head ring tilt is. Although additional computer systems can be used to correct this geometrical deviation, they are usually expensive, time-consuming and require complex procedures $[11-14]$. The other solution to this problem relies on the production of reformatted images in the plane and parallel to the plane containing the intercommissural line, allowing precise localization of a target. Existing methods usually employed 2$D$ rotation transformation to correct the localization error due to the head ring tilt. Traditional correcting algorithm can be expressed by

$$
P^{\prime}=\left[\begin{array}{cc}
\cos \theta & \sin \theta \\
-\sin \theta & \cos \theta
\end{array}\right] P+T
$$

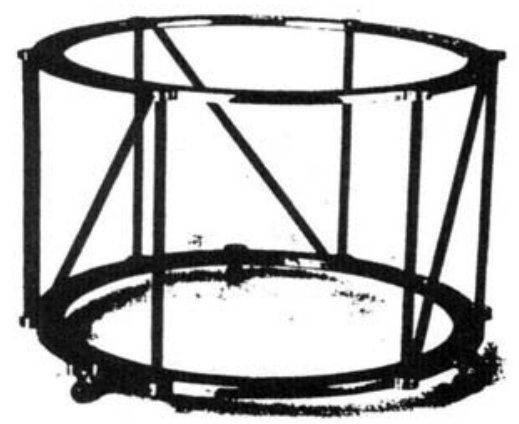

Fig. 1 A CT-compatible headholder (The BRW frame) where $P$ and $P^{\prime}$ are the points before and after transformation respectively, $\theta$ is the orientation and $T$ is the translation vector. However, Eq. (1) cannot provide good estimation to recover the ring rotation error, especially for larger tilt angle. Based on 3-D geometric transformation, we develop a novel algorithm to transform image plane to parallel to the plane containing the intercommissural line, allowing precise localization of a target. The method proposed here can be applied to most CT scanner without complex adjustment. This merit makes it more feasible than other approaches, which required expensive cost or sophisticated procedures.

The remainder of the paper is organized as follows: Section 2 briefly describes the fundamental of the CT localizer used in this approach. Section 3 illustrates the proposed algorithm of correcting localization error using 3-D geometric transform based on the intercommissural line and the head ring tilt angle. Section 4 presents the system setup and shows the good experimental results of the proposed algorithm. The conclusion is included in the final section.

\section{FUNDAMENTAL OF CT LOCALIZER}

In general, the stereotactic system includes a CTcompatible headholder (an aluminum base ring, as shown in Fig. 1), a CT-localizing system, and arcquadrant stereotactic frame [10]. The head ring fits in a CT adaptation plate, which fits on the table of a CT scanner. The frame is a rigid structure that is fixed to the patient's head during both imaging and surgery to define a consistent coordinate system within which to localize targets. There are a series of interchangeable fiducial marker plates exist for CT scanner and are attached during imaging while the frame is in place on the patient's head.

The CT fiducial marker plate contains an $\mathrm{N}$ shaped series of bars, when imaged axially, creates three collinear points. Thus, a CT image consists of

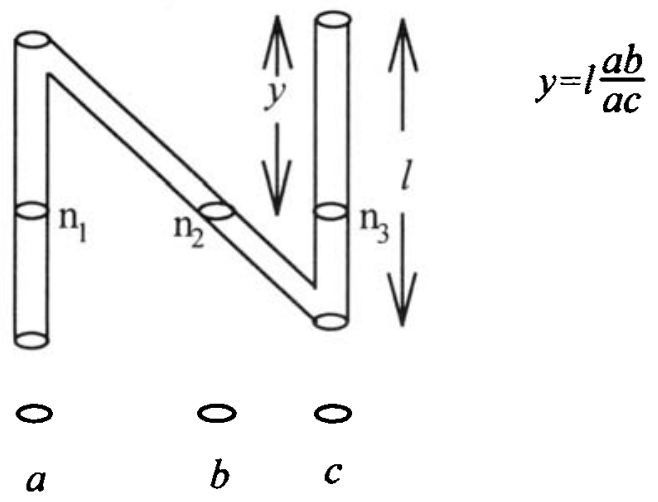

Fig. 2 CT fiducial marker geometry. 
nine such points from the cross section of three plates and allows the 3-D frame coordinates of each middle marker to be determined using geometrical transform (see Fig. 2). That is, the relative location of the middle marker between the vertical posts can be obtained as $y=l \frac{\overline{a b}}{\overline{a c}}$, and the relationship between frame coordinate system and image coordinate system (see Fig. 3) can then be written as

$$
\left[\begin{array}{l}
x \\
y \\
z
\end{array}\right]=\lambda\left[\begin{array}{l}
u \\
v \\
1
\end{array}\right]
$$

where $\lambda=\left[\lambda_{1}, \lambda_{2}, \lambda_{3}\right]^{\mathrm{T}}$ is a $3 * 3$ transformation matrix and $\lambda_{1}=\left[\lambda_{11}, \lambda_{12}, \lambda_{13}\right], \lambda_{2}=\left[\lambda_{21}, \lambda_{22}, \lambda_{23}\right], \lambda_{3}=\left[\lambda_{31}, \lambda_{32}, \lambda_{33}\right]$. Obviously, if nine points $n_{1}, n_{2}, \ldots, n_{9}$ with the frame coordinates $\left(x_{i}, y_{i}, z_{i}\right), i=1 . .9$, and the corresponding image coordinates $\left(\mathrm{u}_{\mathrm{i}}, \mathrm{v}_{\mathrm{i}}, 1\right), \mathrm{i}=1 . .9$ respectively, are given; it is easy to derive $\lambda$ by Eq. (2).

In fact, this transformation matrix, $\lambda$, can be obtained by commercial CT software if the stereotactic procedure is properly performed. Specifically, the 10calization formula for a head ring depends on precisely defining intercommissural line AC-PC as the basis to calculate the required transformation matrix. However, if the head ring tilt occurs during the stereotactic computed tomography scanning, the accuracy of localization is consequent affected. The proposed solution to correct this deviation is the aim of this paper and will be described in next section.

\section{THE PROPOSED ALGORITHM FOR IMPROVING THE LOCAL- IZATION ACCURACY}

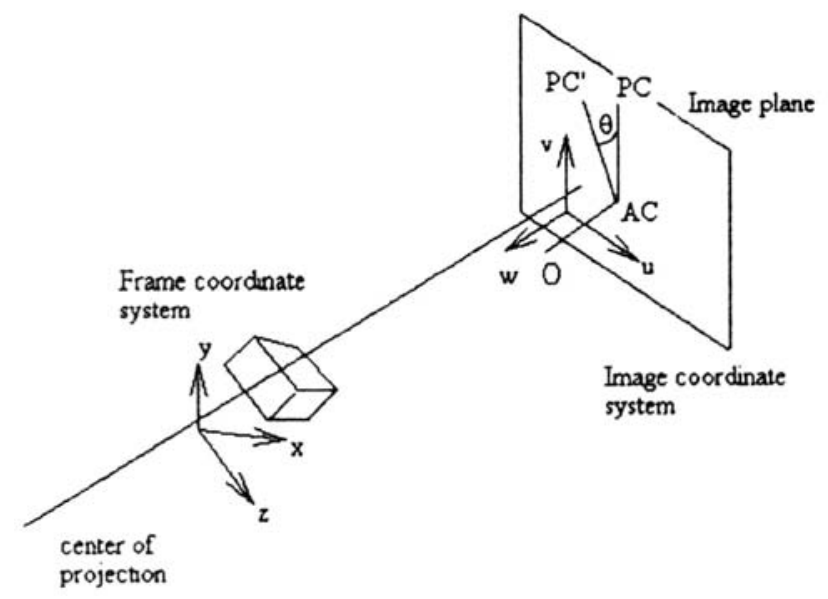

Fig. 3 The frame and image coordinates systtems.
As depicted in Fig. 3, two coordinate systems used in this approach are frame coordinate system (X$\mathrm{Y}-\mathrm{Z}$ ) and image coordinate system (u-v-w), both defined by Cartesian coordinate systems, respectively. A 3-D target point $P$ with its frame coordinates $(x, y, z)$ is related to its corresponding image point $(u, v, 1)$ by Eq.(2).

When the head ring and localizer are aligned to the CT scanner gantry, the coordinate transformation can take a much simpler form in the AP (i.e., $X$-axis), lateral (i.e., Y-axis), and vertical (i.e., Z-axis) coordinates, which can be simply read off from a graphical calculation or from simple distances from the scanner console without need of a complex mathematical transformation. Let the frame coordinates of point $P C$ and $A C$ (two end points of intercommissural line) be $\left(X_{P C}\right.$ $\left.Y_{P C}, Z_{P C}\right)$ and $\left(X_{A C}, Y_{A C}, Z_{A C}\right)$, respectively. Ideally, all these frames coordinates are directly obtained from the CT scan matrix if the intercommissural line is parallel to the v-axis of the image plane. In this case, we have $\left(X_{P C}, Y_{P C}, Z_{P C}\right)=\left(X_{A C}+D, Y_{A C}, Z_{A C}\right)$, where $D=\|\overrightarrow{A C P C}\|,\|\|$ denotes the Euclidean distance. In practice, due to the head ring tilt, the actual intercommissural line rotates an angle $\theta$ from the ideal intercommissural line. Assume its end point $P C$ is changed to point $P C^{\prime}$ with coordinates $\left(X_{P C}^{\prime}, Y_{P C}^{\prime}, Z_{P C}^{\prime}\right)$. Based on the geometric invariant, we have $\|\overline{A C P C}\|=\left\|\overline{A C P C^{\prime}}\right\|=D$, where $D=\sqrt{\left(X_{P C}-X_{A C}\right)^{2}+\left(Y_{P C}-Y_{A C}\right)^{2}+\left(Z_{P C}-Z_{A C}\right)^{2}}$ and the frame coordinates of point $P C$ is consequently obtained. Using the coordinates of point $P C, P C^{\prime}$ and $A C$, for each point $P(X, Y, Z)$ obtained from its ideal image coordinates, a correcting strategy is then carried out to determine its actual location (i.e. $P^{\prime}\left(X^{\prime}, Y^{\prime}, Z^{\prime}\right)$ ) in frame coordinate system. This correcting strategy consists of three steps:

1) Translate $P$ to point $A C$, (i.e., $T_{P \rightarrow A C}$ )

2) Rotate about the normal vector of the plane $P C-A C$ $P C^{\prime}$, (i.e. $\left.R_{P C-A C^{\prime}-P C^{\prime}}\right)$

3) Translate point $A C$ to $P^{\prime}$. (i.e., $T_{A C^{\prime} \rightarrow P^{\prime}}$ ).

The detailed formula is written as

$\left[\mathrm{X}^{\prime}, \mathrm{Y}^{\prime}, \mathrm{Z}^{\mathrm{t}}, 1\right]^{\prime}=T_{A C \rightarrow P^{\prime}} \cdot R_{P C-A C^{-}-P C^{\prime}} \cdot T_{P \rightarrow A C^{\prime}} \cdot[\mathrm{X}, \mathrm{Y}, \mathrm{Z}, 1]^{\mathrm{s}}$ 


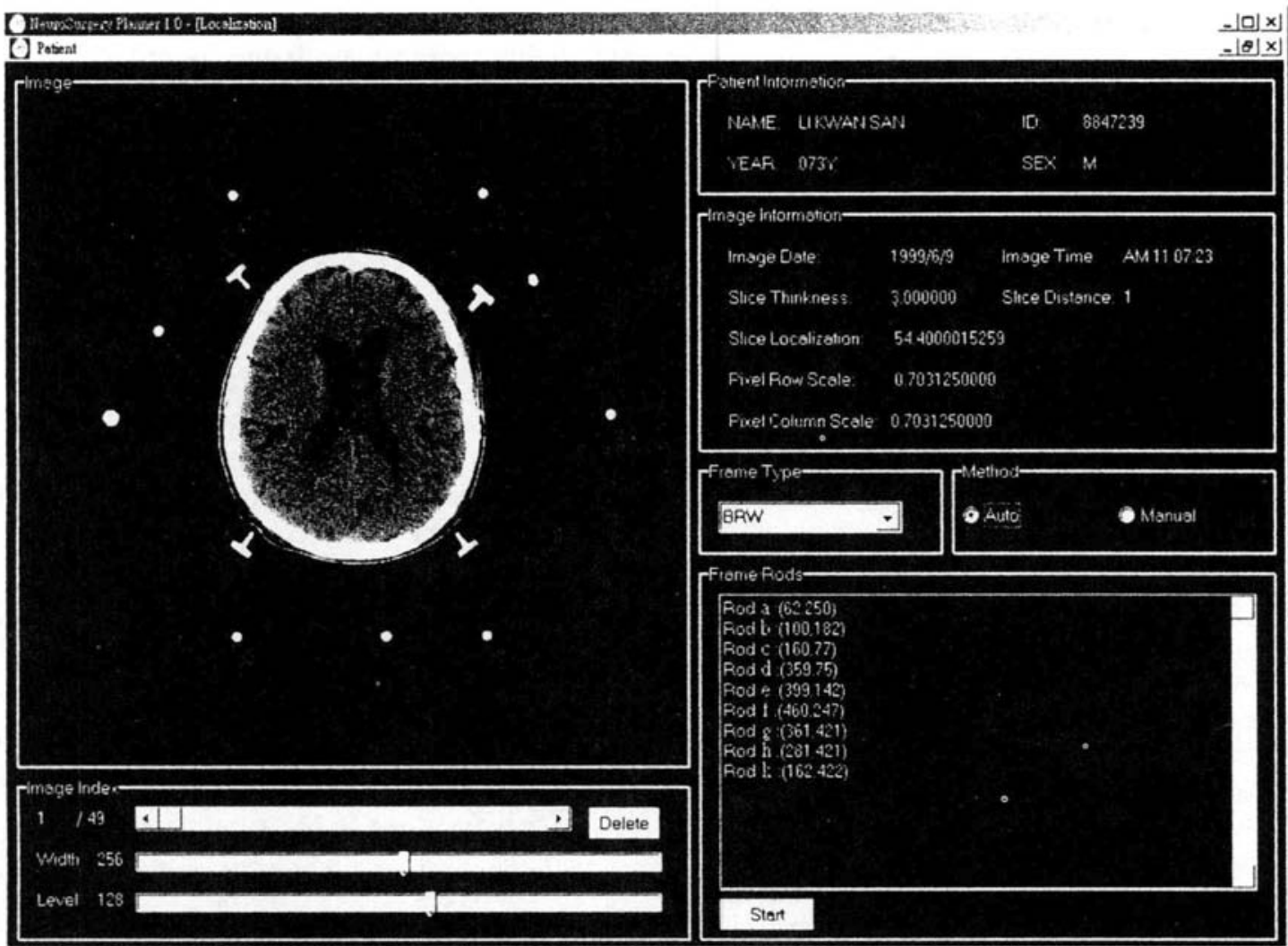

Curront Poal:(016,171)

Fig. 4 Graphic user interface (GUI) of the 3-D localization module in Brain Deep Tracer

Table 1: Comparison of localization performance (i.e., CT scan thickness is $1.5 \mathrm{~mm}$ and interscan spacing is 1.0 mm)

\begin{tabular}{|c|c|c|c|c|c|c|}
\hline \multirow[t]{2}{*}{ Degree } & \multicolumn{3}{|c|}{ Conventional method, mean error $(\mathrm{mm})$} & \multicolumn{3}{|c|}{ The proposed method, mean error $(\mathrm{mm})$} \\
\hline & $\mathrm{x}$ & Y & Z & $\mathrm{X}$ & Y & $\mathrm{Z}$ \\
\hline 3 & 0.70 & 1.28 & 3.30 & 0.65 & 0.94 & 1.65 \\
\hline 5 & 0.92 & 0.97 & 2.14 & 0.85 & 0.87 & 1.07 \\
\hline 10 & 0.61 & 0.68 & 2.08 & 0.52 & 0.55 & 1.05 \\
\hline
\end{tabular}

$$
\text { where } \begin{aligned}
T_{P \rightarrow A C} & =\left[\begin{array}{cccc}
1 & 0 & 0 & -\mathrm{X}_{A C} \\
0 & 1 & 0 & -\mathrm{Y}_{A C} \\
0 & 0 & 1 & -\mathrm{Z}_{A C} \\
0 & 0 & 0 & 1
\end{array}\right], \\
T_{A C \rightarrow P} & =\left[\begin{array}{lllc}
1 & 0 & 0 & \mathrm{X}_{A C} \\
0 & 1 & 0 & \mathrm{Y}_{A C} \\
0 & 0 & 1 & \mathrm{Z}_{A C} \\
0 & 0 & 0 & 1
\end{array}\right],
\end{aligned}
$$

$$
R_{F C-A C-P C}=\left[\begin{array}{cccc}
R_{11} & R_{12} & R_{13} & 0 \\
R_{21} & R_{22} & R_{23} & 0 \\
R_{31} & R_{32} & R_{33} & 0 \\
0 & 0 & 0 & 1
\end{array}\right],
$$

The derivation of the coefficients in the matrixes can be found in reference [15]. Since the derivation is geometric, the computation cost is largely reduced and the localization accuracy is considerably improved. 


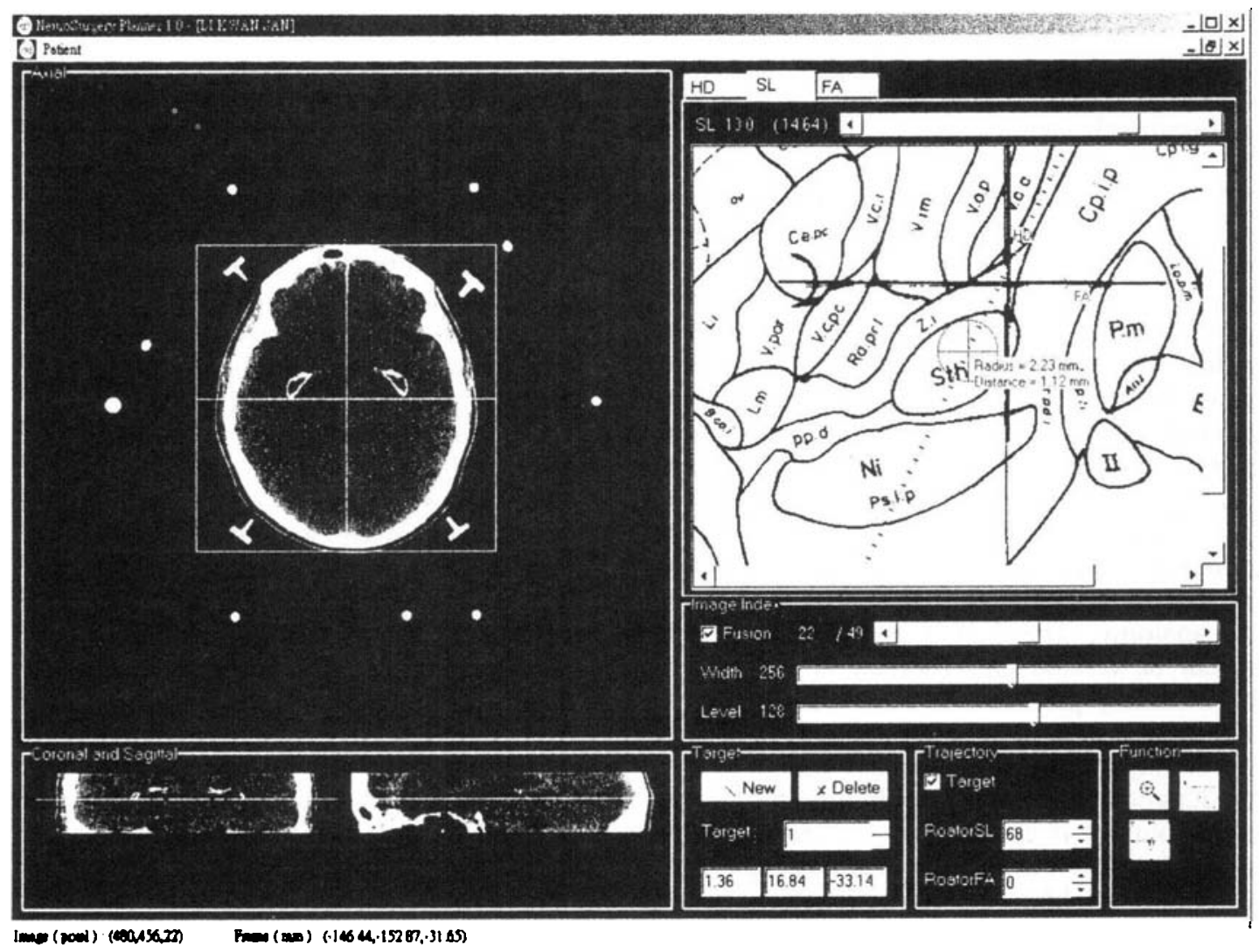

Fig. 5. The GUI of Trajectory Planning module in Brain Deep Tracer.

Table 2: Comparison of localization performance. (i.e., CT scan thickness is $1.5 \mathrm{~mm}$ and interscan spacing is $3.0 \mathrm{~mm}$ )

\begin{tabular}{|c|c|c|c|c|c|c|}
\hline \multirow[t]{2}{*}{ Degree } & \multicolumn{3}{|c|}{ Conventional method, mean error $(\mathrm{mm})$} & \multicolumn{3}{|c|}{ The proposed method, mean error (mm) } \\
\hline & $\mathrm{x}$ & $\mathrm{Y}$ & $\mathrm{Z}$ & $\mathrm{x}$ & Y & Z \\
\hline 1 & 1.68 & 2.00 & 3.30 & 1.59 & 1.75 & 1.65 \\
\hline 3 & 1.67 & 2.28 & 2.97 & 1.52 & 1.82 & 1.49 \\
\hline 5 & 0.94 & 3.68 & 5.34 & 0.61 & 1.97 & 2.69 \\
\hline 7 & 2.04 & 1.65 & 3.83 & 1.91 & 0.77 & 1.95 \\
\hline 10 & 1.98 & 2.10 & 4.99 & 1.55 & 1.52 & 2.57 \\
\hline
\end{tabular}

\section{SYSTEM SETUP AND EXPERI- MENTAL RESULTS}

Since early-generation CT scanners lacked precision table movement in the vertical, it was required to establish the relationship of three points in each scan image to the reference system to ensure the spatial accuracy between 3-D coordinates defined on different CT scan images. But new-generation CT scanners improved the precision of the mechanical movement of the scanner table and gantry, the vertical coordinate accuracy increased significantly. These mechanical advances, coupled with computer software advances such as rapid reformatting of $\mathrm{CT}$ scans and volume rendering, suggested a simplified method of defining a 3-D coordinate system. However, the traditional stereotactic software cannot compensate the localization error due to the head ring tilt. To overcome the drawback of previous systems, we develop a stereotactic software system, named Brain Deep Tracer. This system can help a neurosurgeon to perform the miniinvasive hrain surgery e.g. Functional Stereotaxy, and the diagnosis of brain disease such as Parkinson's. 
General speaking, this software system includes a rich set of functions such as 3-D reconstruction of brain atlas, 3-D localization of patient's brain CT data, registration of brain atlas and CT data, fusion of brain atlas and CT data, and surgical trajectory planning. More important, this system can correct the distortion due to the tilt of head frame and make the localization result more accurate.

Brain Deep Tracer allows the neurosurgeon to determine the entry point and target, and tographically follow the probe's pathway through all the tissue. By reviewing all slices to be intersected, the neurosurgeon can assess the entire trajectory and ensure avoidance of critical structures including vessels and the cerebral ventricles. Probe trajectories can be seen on orthogonal or oblique slices to facilitate developing the best plan. The stereotactic arc is also graphically displayed, showing exactly how it would appear in relation to the patient's anatomy. The 3-D display of Brain Deep Tracer can be rotated and zoomed in real-time for viewing from any perspective. The neurosurgeon can instantly change the entry point and trajectory to the target by simply modifying the arc angle settings.

For functional stereotaxy, including thalamotomy and pallidotomy, it is essential to determine precisely the optimal target point and the best path to reach it. The options for the diagnosis and treatment of brain lesions have been greatly increased by pairing stereotactic surgery with modem diagnostic imaging such as CT or MR image. Stereotactic localization with the aid of CT or MR is widely employed to direct a variety of neurosurgical procedures in critical areas of the central nervous system. One of the primary benefits of stereotaxy is the precision with which lesions can be localized, and this accuracy with makes techniques such as radiosurgery feasible.

Before the surgical operation, the neurosurgeon must fix the patient on the BRW localization frame, which consists of nine carbon rods. The diameters are $10 \mathrm{~mm}$ for one rod and $6 \mathrm{~mm}$ for the remaining eight. The rods are arranged in three $\mathrm{N}$-shaped formations of three oblique and six straight rods. This frame is fixed to the skull and served as an immovable reference $[16,17]$.

Since intracranial targets are not directly visible from outside, they must be deduced from radiological images like CT image or MR image. MRI provides a better visualization of soft brain tissue compared with CT, whereas CT provides excellent geometrical accuracy of the image. In this system, we encourage the neurosurgeon to use CT image for the sake of increasing the accuracy of localization.

After CT scanning, the actual patient's data, read as DICOM CT images, are imported into our system, interpolated, and their axial, coronal, sagittal views are generated. The 9 rods will be displayed on the CT image as small circles. Using the centroids of these 9 cir- cles, a stereotactic localization algorithm is derived by Eq. (2) and the target with CT-based coordinates can then be transferred to its corresponding frame-based coordinates for the surgical operation later. Fig. 4 is the graphic user interface (GUI) of the 3-D localization module in Brain Deep Tracer. In this figure, the crosssections of 9 rods of the BRW localization frame are displayed on the CT image as small circles, denoted as $a, b, c, d, e, f, g, h$, and $k$. In the GUI, the patient's information is shown in top of the right part and the $x, y$ coordinates of point $a, \ldots, k$ are shown in the bottom of the right part.

The localization system produces reference marks on CT slices, which are used to calculate stereotactic coordinates for specified points digitized from CT scans. The coordinates of four simulated intracranial target points within a scanned phantom with different CT scan thickness $(1.5 \mathrm{~mm}$ and $3.0 \mathrm{~mm})$ and interscan spacing $(1.0 \mathrm{~mm}$ and $3.0 \mathrm{~mm})$ were calculated using conventional 2-D correcting method and the proposed algorithm. Tables 1-4 illustrate the comparison of localization accuracy using conventional method and our algorithm under different tilt angle $\theta$. The range of $\theta$ is set to be in the range $[0 \ldots 10]$. This assumption is reasonable because that the seriously head ring tilt seldom occur when the surgery is performed by an experienced neurosurgeon. From these tables, it is observed that clearly significant improvements in performance have been made by the proposed approach. Moreover, this technique can easily be utilized to stereotactic magnetic resonance imaging.

In summary, there was significant difference of localization error across different interscan spacing and scan thickness. The mean error decreased as the interscan spacing decreased, indicated that more CT scans obtained through the lesion, the more accurate is localization. In addition, accuracy can also be improved as scan thickness decreased by reducing the effect of partial averaging. However, if the scan thickness is decreased, the total number of CT scans required to view the volume of the lesion is consequently increased, resulting in higher radiation exposure. Thus, it is a good choice that for small lesions, the smallest scan thickness and interscan spacing are employed to obtain the maximum accuracy. On the other hand, for larger lesions, larger scan thickness and interscan spacing are used without loss of accuracy. For lesions needed extreme accuracy, it is recommended [14] that angiography is the best choice, although that it fails to represent the true 3-D nature of the lesion. As yet, there are twenty patients using our CT localization techniques followed by electrophysiologic confirmation of the target site. No complications of either the imaging or the surgical parts of the procedures have occurred in postoperative analysis to these patents undergoing the neurosurgery. 
Table 3: Comparison of localization performance. (i.e., CT scan thickness is $3.0 \mathrm{~mm}$ and interscan spacing is $1.0 \mathrm{~mm}$ )

\begin{tabular}{|c|c|c|c|c|c|c|}
\hline \multirow[t]{2}{*}{ Degree } & \multicolumn{3}{|c|}{ Conventional method, mean error $(\mathrm{mm})$} & \multicolumn{3}{|c|}{ The proposed method, mean error $(\mathrm{mm})$} \\
\hline & $\mathrm{X}$ & Y & $\mathrm{Z}$ & $\mathrm{X}$ & $\mathrm{Y}$ & $\mathrm{Z}$ \\
\hline 1 & 1.38 & 1.32 & 3.76 & 1.35 & 0.56 & 1.88 \\
\hline 3 & 0.90 & 2.84 & 5.57 & 0.75 & 1.72 & 2.79 \\
\hline 5 & 1.59 & 1.30 & 2.28 & 1.47 & 0.83 & 1.15 \\
\hline 7 & 0.81 & 0.58 & 3.83 & 0.72 & 0.44 & 1.95 \\
\hline 10 & 0.86 & 0.62 & 4.13 & 0.79 & 0.35 & 2.13 \\
\hline
\end{tabular}

Table 4: Comparison of localization performance (i.e., CT scan thickness is $3.0 \mathrm{~mm}$ and interscan spacing is $3.0 \mathrm{~mm}$ )

\begin{tabular}{|c|c|c|c|c|c|c|}
\hline \multirow{2}{*}{ Degree } & \multicolumn{3}{|c|}{ Conventional method, mean error (mm) } & \multicolumn{3}{|c|}{ The proposed method, mean error $(\mathrm{mm})$} \\
\hline & $X$ & Y & $\mathrm{Z}$ & $\mathrm{X}$ & $\mathrm{Y}$ & Z \\
\hline 1 & 1.73 & 1.57 & 4.30 & 1.65 & 1.56 & 2.15 \\
\hline 3 & 2.08 & 3.83 & 3.49 & 1.85 & 2.72 & 1.75 \\
\hline 5 & 1.84 & 2.74 & 4.47 & 1.52 & 1.95 & 2.25 \\
\hline 7 & 1.84 & 1.78 & 6.19 & 1.52 & $\overline{1.44}$ & 3.15 \\
\hline 10 & 2.54 & 3.77 & 4.91 & 1.85 & 2.35 & 2.53 \\
\hline
\end{tabular}

\section{CONCLUSIONS}

Recently, computerized tomography becomes a useful tool for stereotactic localization due to its low risk, high resolution, ease to use, and ability to be modified to increase accuracy. Using standard CT software, the plane of the intercommissural line cannot be predicated from the external anatomy of the skull due to the head ring tilt. Therefore, a novel approach using 3-D geometric transform is proposed to correct this location deviation in the procedure of stereotaxic localization. The algorithm increases the convenience and flexibility of frame-based stereotactic guidance by providing a means of noninvasive localization that can be accomplished selectively at a separate time from the guidance part of a stereotactic operative procedure. Experimental results also confirm the effectiveness of the proposed method. This technique has been implemented in a novel stereotactic software system, named Brain Deep Tracer. With the valuable functions such as fusion of brain atlas and CT data, and surgical trajectory planning, the Brain Deep Tracer has been successfully applied to clinic application for neurosurgery in Chang Gung Memorial Hospital.

\section{ACKNOWLEDGEMENT}

The authors would like to thank the National Science Council of the R.O.C. for financial support of this manuscript under contract No. NSC90-2212-E182-006.

\section{REFERENCES}

1. C. A. Gleason, B. L. Wise and B. Feinstein, "Stereotactic localization with computerized tomographic scanning, biopsy and radiofrequency treatment of deep brain lesions", Neurosurgery, vol. 2, pp. 217-222, 1978.

2. R. A. Brown, "A computerized tomographyComputer graphics approach to stereotactic localization", J. Neurosurgery, vol. 50, pp. 715-720, 1979.

3. W. Huk and U. Baer, A new targeting device for stereotaxic procedures within the CT scanner, Neuroradiology, vol. 19, pp. 13-17,1980.

4. M. L. Rhodes, W. V. Glenn, Y. W. Azzawi and R. Slater, "Stereotactic neurosurgery using 3-D image data from computed tomography", J. Med Syst. Vol. 6, pp. 105-119, 1982.

5. R. E. Latchaw, L. D. Lunsford and W. H. Kennedy, "Reformatted imaging to define the intercommissural line for CT-guided stereotaxic functional neurosurgery", AJNR, vol. 6, pp. 429-433, 1985.

6. L. D. Lunsford, "Innovations in stereotaxic technique coupled with computerized tomography", Contemp Neurosurg, vol. 4, pp. 1-6, 1982.

7. T. L. Hardy, J. Koch and A. Lassiter, "Computer graphics with computerized tomography for functional neurosurgery", Appl. Neurophysiol vol. 46, pp. 217-226, 1983.

8. P. Grunert, J. Maurer, W. Muller-Forell, “Accuracy of Stereotactic Coordinate Transformation using a 
Localisation Frame and Computed Tomographic Imaging", Neurosurg Rev, Vol. 22, pp.173-187, 1999.

9. Yulong Yan, Xudong Bao, Yi Bai, Limin Luo, Huazhong Shu, Yao Fu, “ 3-D Imaging and Stereotactic Radiosurgery, “ IEEE Engineering in Mediciene and Biology, pp. 47-52, July/August, 1997.

10. Patrick J. Kelly, Tumor stereotaxis, W. B. Saunders Company, 1991.

11.K. R. Winston and W. Lutz, "Linear accelerator as a neurosurgical tool for stereotactic radiosurgery," Neurosurgery, vol. 22, pp. 454-463, 1988.

12.R. D. Bucholz, H. W. Ho and J. P. Rubin, "Variables affecting the accuracy of stereotactic localization using computerized tomography", J. Neurosurgery, vol. 79, pp. 667-673, 1993.

13.T. Asakura, K. Uetsuhara and R. Kanemaru, "An applicability study on a CT-guided stereotactic technique for functional neurosurgery", Appl. Neu- rophysiol, vol. 48, pp. 73-76, 1985.

14. M. P. Heilbrun, S. Koehler, P. Macdonald, V. Diemionow and $\mathrm{W}$. Peters, "Preliminary experience using an optimized three-point transformation algorithm for spatial registration of coordinate systems: a method of noninvasive localization using frame-based stereotactic guidance systems", J. Neurosurgery, vol. 81, pp. 676-682, 1994.

15.D. F. Rogers, Procedural elements for computer graphics, McGraw-Hill, New-York, 1985.

16.P. Grunert, J. Maurer, W. Muller-Forell, "Accuracy of Stereotactic Coordinate Transformation using a Localisation Frame and Computed Tomographic Imaging", Neurosurg Rev, Vol.22, pp.173-187, 1999.

17. Birg W, Mundinger F, "Direct target point determination for stereotactic brain operations from CT data and the calculation of setting parameters for polar coordinate stereotactic devices." Appl Neurophsiol, Vol 45, pp. 387-395, 1982. 